\title{
Multiple Moving Targets Detection and Parameters Estimation in Strong Reverberation Environments
}

\author{
Ge Yu and Shengchun Piao \\ Acoustic Science and Technology Laboratory and College of Underwater Acoustic Engineering, Harbin Engineering University, \\ Harbin, China \\ Correspondence should be addressed to Ge Yu; liz.221@163.com
}

Received 30 June 2016; Accepted 30 August 2016

Academic Editor: Mario Terzo

Copyright (C) 2016 G. Yu and S. Piao. This is an open access article distributed under the Creative Commons Attribution License, which permits unrestricted use, distribution, and reproduction in any medium, provided the original work is properly cited.

\begin{abstract}
This paper considers the problem of multiple moving targets detection and parameters estimation (direction of arrival and range) in strong reverberation environments. As reverberation has a strong correlation with target echo, the performance of target detection and parameters estimation is significantly degraded in practical underwater environments. In this paper, we utilize two uniform circular arrays to receive plane wave of the linear frequency modulation signal reflected from far-field targets. On the basis of received signal, we build a variance matrix of multiple beams by using modal decomposition, conventional beamforming, and fractional Fourier transform (FrFT). We then propose a novel detection method and an estimation method of parameters based on the constructed image. A significant feature of the proposed methods is that our design does not involve any a priori knowledge about targets number and parameters of marine environments. Finally, we demonstrate via numerical simulation examples that the detection probability and the accuracy of estimated parameters of the proposed method are higher than the existing methods in both low signal-to-reverberation ratio and signal-to-noise ratio environment.
\end{abstract}

\section{Introduction}

Targets detection and associated parameter estimation such as range, velocity, and direction of arrival (DOA) have various practical use applications in both military and civil. However, in shallow environments, reverberation as the main interference is generated by multiple reflection and diffusions of transmitted signal from rough boundaries interfaces (surface and bottom reverberation) [1]. The producing mechanism of reverberation means that it is highly correlated with target echo. As a result, it is very difficult to find a desired signal in the reverberation environment, especially when the signalto-reverberation ratio (SRR) is relatively low. Therefore, we can draw a conclusion that reverberation results in high probability of false alarm and low detection probability in severe reverberation environment.

In the case of reverberation, some methods are used to enhance SRR. Cheepurupalli and Konduri combined empirical mode decomposition (EMD) with adaptive boosting (AdaBoost), which is a learning algorithm, to process the echo signal in reverberation environment [2]. And as a result of AdaBoost, the accuracy and robustness of targets detection method are improved significantly. Similarly, the principle component inverse (PCI) algorithm has also been used to detect targets in sonar system. It is utilized to separate targets from the received signals and to eliminate the reverberation. With PCI algorithm, Ginolhac and Jourdain presented a method, which combined with beamforming, to resolve the rank problem and to get a better separation [1]. To make use of spatial information, matched-field processing (MFP) is developed to localize targets in range, depth, and azimuth [3]. It is a spatial-filtering technique and can be used in both passive and active sonar systems. Unfortunately, a stratified waveguide model of the acoustic environment is a necessary prior knowledge in this method $[4,5]$. Considering utilizing Doppler shift features of moving target, fractional Fourier transform (FrFT) is widely applied to radial velocity and range estimation [6-8] and it performs well in low signal-to-noise ratio (SNR) condition, whereas the detection probability and accuracy of parameters estimation decrease along with the reduction of SRR.

The methods which we have already listed above are applied by using traditional acoustic pressure sensor system. 


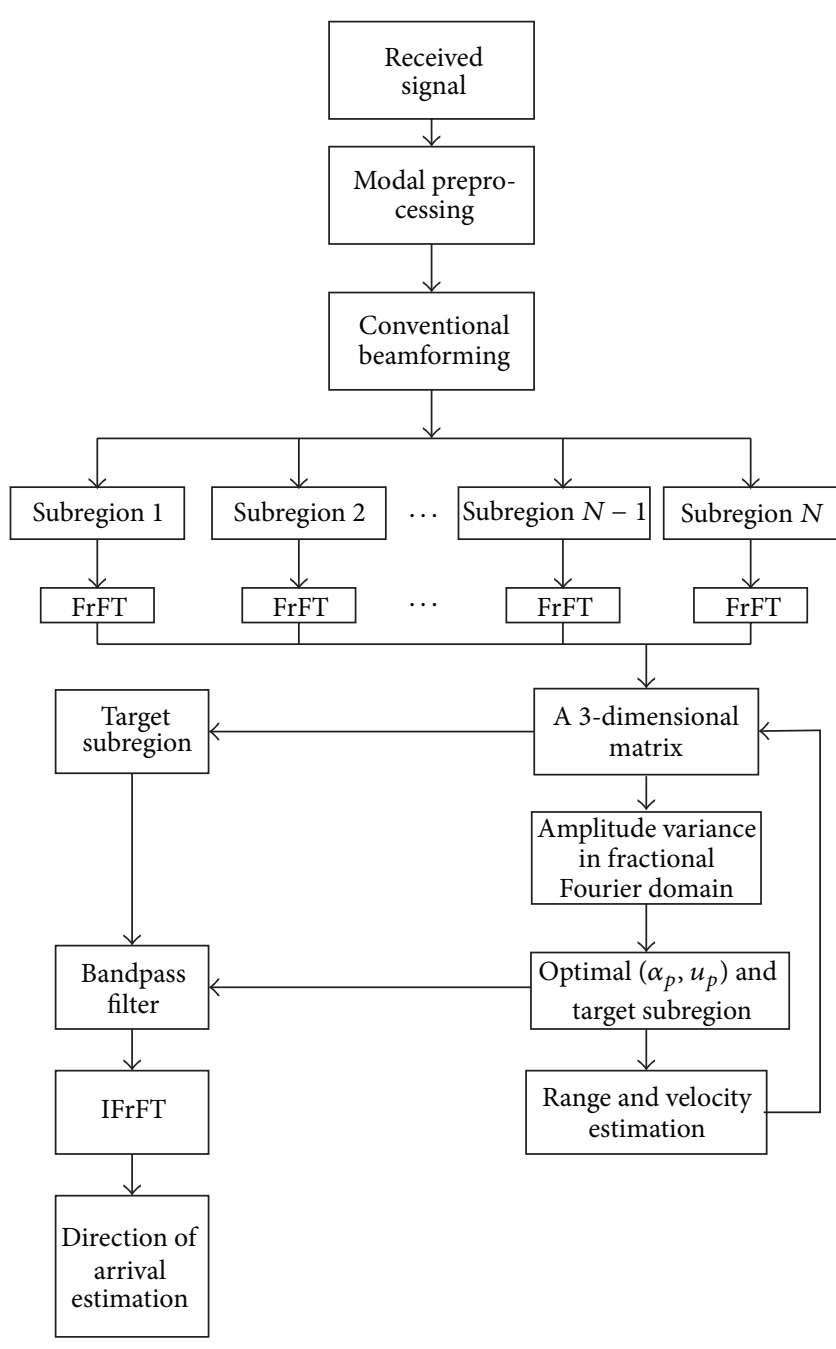

FIGURE 1: The block diagram of the proposed method for detection and parameters (range and direction of arrival) estimation of multiple moving targets.

As acoustic vector array can be used to measure both pressure and particle velocity at a point in space $[9,10]$, in recent years, it has come to receiving particular attention. In particular, the vector sensor array has some characteristics which can bring many benefits such as high resolution and 4-dimensional information (pressure and particle velocities along the three orthogonal directions). Specifically, using a single vector sensor can estimate the azimuth elevation direction of the target [11].

Spurred by the above pioneering works, we aim to develop an effective targets' detection method and a parameters' estimation method with active sonar system in severe reverberation environment. Two uniform circular sensor arrays are used in the proposed method. In the meanwhile, a linear frequency modulated (LFM) signal is used as the transmitted signal due to its high resolution and sensitivity of Doppler shift. The block diagram of the proposed method is shown in Figure 1. For incorporating temporal information, spatial bearing distribution of received signal, and Doppler shift information of multiple moving targets into a new observed matrix, we can construct this new 3-dimensional (3D) matrix by utilizing modal preprocessing, fractional Fourier transform (FrFT), and conventional beamforming to divide the region of interest into $N$ subregions in the signal preprocessing section. Then, in order to utilize the nonstationarity of reverberation, we calculate the changing trends of magnitudes in different subregions in the fractional Fourier domain to detect targets and to get the optimal values and the target subregion. With these optimal values, we can estimate the range and velocity of multiple moving targets. In addition, the target subregion can be exploited to suppress reverberation and get a high SRR value. Hence, the multiple signal classification method (MUSIC) can be applied to estimate the DOA of multiple targets in a high SRR environment. follows:

The main contributions of this paper are summarized as

(i) Compared with the method in [6], the multiple moving targets can be detected in severe environment without any prior knowledge.

(ii) The proposed parameters estimation method can obtain significant performance in extremely low SNR and SRR environments.

(iii) With two uniform circular arrays, the proposed DOA estimation method can overcome the port and starboard ambiguity problem and solve the zero crossings at various different argument values in Bessel functions.

(iv) The detection probability and the precision of parameters estimation are not affected by the number of targets.

The rest of the paper is organized as follows. The models of the received signals and active sonar system are given in Section 2. In Section 3, signal preprocessing is introduced including modal preprocessing, Dolph-Chebyshev beamforming, and fractional Fourier transform. How to detect the moving target is presented in Section 4. Detailed discussions of parameters estimation are provided in Section 5 . Simulation results and discussion are shown in Section 6. Conclusions and future work are drawn in the last section.

We use the following notations in this paper: $\|\cdot\|$ denotes the vector norm. $[\cdot]^{\#}$ represents the Moore-Penrose pseudoinverse. $[\cdot]^{T}$ denotes vector or matrix transpose.

\section{Active Sonar System Model}

In this paper, we have some assumptions to build the active sonar system model. First, we assume that the targets are located in the far field of the sensor array. Consequently, plane wave is used to represent target echo; second, the Gaussian noise at each sensor is stationary and temporally; third, the reverberation is locally stationary.

With these assumptions, an active sonar system is established as shown in Figure 2. The received array is made up of two uniform circular arrays. Particularly, each array has $Q$ sensors with radius $R_{1}$ and $R_{2}\left(R_{1}>R_{2}\right)$, respectively, and the $q$ th sensor of $i$ th array located at $\mathbf{h}_{q i} \equiv\left(\left\|\mathbf{h}_{q i}, \varphi_{q i}\right\|\right)$, where 


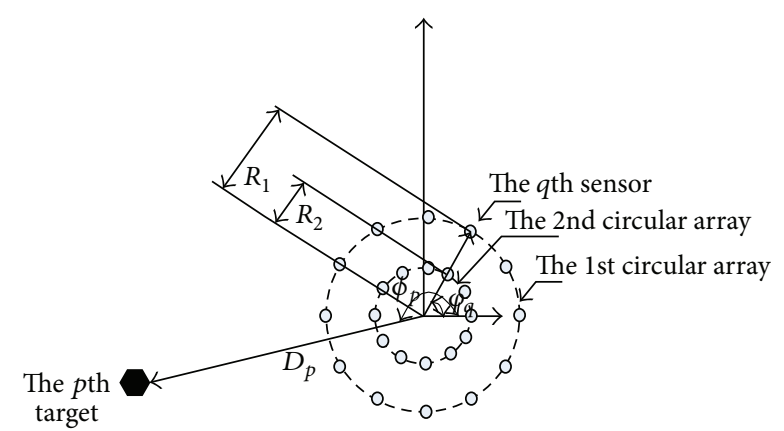

FIGURE 2: Geometry of two uniform circular arrays and targets.

$q=1, \ldots, Q$ and $i=1,2$ in the polar coordinates system. Also, there are $P$ targets located at $\mathbf{u}_{p}$, with $\left\|\mathbf{u}_{p}\right\|=D_{p}$ denoting the distance between the $p$ th target and origin of the array and $\phi_{p}$ is the incident angle of the $p$ th target; $\widehat{\mathbf{u}}_{p}=$ $\mathbf{u}_{p} /\left\|\mathbf{u}_{p}\right\|$ is a unit vector along the direction of the $p$ th target.

In reverberation environment, the received signal $x_{q i}(t)$ of the $q$ th sensor in the $i$ th array at time $t$ can be described as

$$
x_{q i}(t)=\sum_{p=1}^{P} s_{p}\left(t+\tau_{p q i}\right)+n_{q i}(t)+r_{q i}(t)
$$

where $\tau_{p q i}=\mathbf{h}_{q i} \cdot \mathbf{u}_{p} / c$ denotes the propagation delay of the echo from the $p$ th target between the $q$ sensor and the origin in the $i$ th array and $s_{p}(t)$ is the $p$ th target echo at the origin of the circular arrays. The expression of $s_{p}(t)$ is

$$
s_{p}(t)=\gamma_{p} s\left(\sigma_{p} t-\tau_{p}\right),
$$

where $\sigma_{p}$ is the time compression/stretching parameter; $\tau_{p}$ is the time delay; and $\gamma_{p}$ is the range-dependent attenuation. $n_{q i}$ is additive white Gaussian additive noise at the $(q, i)$ sensor which is assumed to be a zero-mean complex random process. $r_{q i}$ is reverberation which is considered as a sum of undesirable echoes issued from the transmitted signal received at the $(q, i)$ sensor.

As reverberation signal is dependent on the transmitted signal, it is closely bound up with target echo signal. Our scheme in this paper is to overcome the effects of reverberation and address the problem of estimating the range and DOA of multiple moving targets.

\section{Signal Preprocessing}

In the proposed method, LFM signal is used as transmitted signal due to the detection sensitivity and the high range resolution. However, some methods such as classical delay-and-sum beamforming and MUSIC are not applicable resulting from the wideband characteristics. In this section, we use three steps of signal preprocessing: Step 1: modal preprocessing [6] is used to convert the echo signals into different spatial orthogonal modes; Step 2: we divide the spatial region of interest into several subregions to obtain the spatiotemporal information by virtue of Dolph-Chebyshev beamforming; Step 3: fractional Fourier transform (FrFT) is used to transform the received signal from frequency domain to fractional Fourier domain. With the help of these three signal processing methods, we finally reconstruct a 3-dimensional matrix, which contains spatial information, temporal information, and Doppler shift information of different moving targets.

3.1. Modal Preprocessing. In this subsection, we firstly use Fourier transform to convert the received signals from time domain to frequency domain. Then, (1) can be represented as

$$
X_{q i}(\omega)=\sum_{p=1}^{P} S_{p}(\omega) e^{j \omega \tau_{p q i}}+\mathcal{N}_{q i}(\omega)+\mathscr{R}_{q i}(\omega),
$$

where $X_{q i}(\omega), S_{p}(\omega), \mathcal{N}_{q i}(\omega)$, and $\mathscr{R}_{q i}(\omega)$ are the Fourier domain expressions of $x_{q i}(t), s_{p}(t), n_{q i}(t)$, and $r_{q i}(t)$, respectively. With Jacobi-Anger expansion of a plane wave [12], $e^{j \omega \tau_{p q i}}$ can be rewritten as

$$
e^{j \omega \tau_{p q i}}=e^{j(\omega / c) \mathbf{h}_{q i} \cdot \mathbf{u}_{p}}=\sum_{m=-M}^{M} J_{m}\left(\frac{\omega}{c} R_{q i}\right) e^{j m\left(\varphi_{q i}+\pi / 2-\phi_{p}\right)}
$$

where $M=\left\lceil\omega / c\left\|R_{1}\right\|\right\rceil$ is the truncation of the infinite series [13]. Thus, the signals received at the sensor arrays can be expressed as

$$
\begin{aligned}
\mathbf{X}(\omega) & =\mathbf{X}_{1}(\omega)+\mathbf{X}_{2}(\omega) \\
& =\mathbf{J}(\omega) \mathbf{A S}(\omega)+\mathbf{N}(\omega)+\mathbf{R}(\omega)
\end{aligned}
$$

where $\mathbf{X}_{i}=\left[X_{1 i}(\omega), X_{2 i}(\omega), \ldots, X_{\mathrm{Q} i}(\omega)\right]^{T}(i=1,2), \mathbf{N}=$ $\left[\mathscr{N}_{11}+\mathcal{N}_{12}, \ldots, \mathcal{N}_{\mathrm{Q} 1}+\mathcal{N}_{\mathrm{Q} 2}\right]^{T}$, and $\mathbf{R}=\left[\mathscr{R}_{11}+\mathscr{R}_{12}, \ldots, \mathscr{R}_{\mathrm{Q} 1}+\right.$ $\left.\mathscr{R}_{\mathrm{Q} 2}\right]^{T}$ are all $\mathrm{Q} \times 1$ matrices; other related matrices are described in the following.

A is a $(2 M+1) \times P$ frequency-independent steering matrix that can be written as

$$
\mathbf{A}=\left[\begin{array}{ccc}
e^{-j M \phi_{1}} & \cdots & e^{-j M \phi_{P}} \\
\vdots & \ddots & \vdots \\
e^{j M \phi_{1}} & \cdots & e^{j M \phi_{P}}
\end{array}\right]
$$

J is a $Q \times(2 M+1)$ matrix shown as

$$
\mathbf{J}=\mathbf{K}\left(\mathbf{L}_{1}(\omega)+\mathbf{L}_{2}(\omega)\right)
$$

where $\mathbf{K}$ of size $Q \times(2 M+1)$ satisfies

$$
\left[\begin{array}{ccc}
e^{-j M\left(\varphi_{1}+\pi / 2\right)} & \cdots & e^{j M\left(\varphi_{1}+\pi / 2\right)} \\
\vdots & & \vdots \\
e^{-j M\left(\varphi_{\mathrm{Q}}+\pi / 2\right)} & \cdots & e^{j M\left(\varphi_{\mathrm{Q}}+\pi / 2\right)}
\end{array}\right]
$$


$\mathbf{L}_{i}$ is a diagonal matrix of size $(2 M+1) \times(2 M+1)$ defined as

$$
\begin{aligned}
& {\left[\begin{array}{llll}
J_{-M}\left(\frac{\omega R_{i}}{c}\right) & & & \\
& J_{-M+1}\left(\frac{\omega R_{i}}{c}\right) & & \\
& & \ddots & \\
& & J_{M}\left(\frac{\omega R_{i}}{c}\right)
\end{array}\right],} \\
& i=1,2 .
\end{aligned}
$$

S is a $P \times 1$ vector which is composed of $P$ target echoes that can be expressed as

$$
\mathbf{S}=\left[\begin{array}{c}
\gamma_{1} S_{1}(\omega) \\
\vdots \\
\gamma_{P} S_{P}(\omega)
\end{array}\right] .
$$

As the location of each sensor is known in advance, the modal preprocessing [6] can be used to process the received signals at the sensor arrays by evaluating

$$
\mathbf{Z}(\omega)=\mathbf{J}^{\#}(\omega) \mathbf{X},
$$

where $\mathbf{J}^{\#}=\left(\mathbf{J}^{T} \mathbf{J}\right)^{-1} \mathbf{J}^{T}$ is the pseudoinverse of $\mathbf{J}$ and $\mathbf{Z}$ is a $(2 M+$ 1) $\times 1$ matrix.

With the above modal preprocessing, we can transform wideband received signals at two circular arrays into signals at different modes. This is beneficial for the following conventional beamforming method.

3.2. Conventional Beamforming. Noting that the two concentric uniform circular arrays are omnidirectional and the modal components of the signals can be combined to form frequency invariant beamformers [14], we construct $L$ beamformers to divide the observing region into $L$ subregions in this subsection. Dolph-Chebyshev patterns are widely adopted when designing beamformer due to the narrow main-lobe and constant side-lobe level.

We set $\mathbf{w}$ as the weight vector; the expression is

$$
\begin{gathered}
\mathbf{w}\left(\theta_{l}\right)=\left[e^{-M j \theta_{l}} I_{-M}, e^{-(M-1) j \theta_{l}} I_{-(M-1)}, \ldots, e^{-j \theta_{l}} I_{-1}, 2 I_{0},\right. \\
\left.e^{j \theta_{l}} I_{1}, \ldots, e^{(M-1) j \theta_{l}} I_{M-1}, e^{M j \theta_{l}} I_{M}\right],
\end{gathered}
$$

where $\theta_{l}$ is the look direction of the $l$ th subregion and $\mathbf{w}$ is a $1 \times$ $(2 M+1)$ vector. $I_{m}$ is the weight of Dolph-Chebyshev pattern given in [15]. It follows from Dolph-Chebyshev beamforming that the output of the $l$ th subregion can be expressed as

$$
\widetilde{\mathbf{b}}\left(\theta_{l}, \omega\right)=\operatorname{diag}\left(\mathbf{w}\left(\theta_{l}\right)\right) \mathbf{Z}(\omega),
$$

where $\widetilde{\mathbf{b}}\left(\theta_{l}, \omega\right)$ is a $(2 M+1) \times 1$ vector. The summation of the magnitude of the signals at $2 M+1$ modes is

$$
b\left(\theta_{l}, \omega\right)=\sum_{n=-M}^{M} \widetilde{\mathbf{b}}_{n}, \quad \text { for } l=1,2, \ldots, L .
$$

With beamformed output equation (14), we construct a new observation vector as

$$
\mathbf{b}(\omega)=\left[b\left(\theta_{1}, \omega\right), \ldots, b\left(\theta_{L}, \omega\right)\right] .
$$

By beamforming, we can acquire the spatial distribution information of received signals. In the following, we employ fractional Fourier transform to obtain the temporal information and Doppler shift information of different targets.

3.3. Fractional Fourier Transform. Note that the fractional Fourier transform (FrFT), which is a generalization of the traditional Fourier transform, is a representation of signals using an orthonormal basis formed by LFM signals. Therefore, the certain angle FrFT of the LFM signals is a delta function. In general, the fractional Fourier transform of signal $x(t)$ with transform angle $\alpha$ is defined as

$$
X_{\alpha}(\mu)=\int_{-\infty}^{\infty} x(t) K_{\alpha}(t, \mu) d t
$$

where $K_{\alpha}(t, \mu)$ is the transformation kernel and satisfies

$$
\begin{aligned}
& K_{\alpha}(t, \mu) \\
& = \begin{cases}\Gamma_{\alpha} e^{j 2 \pi\left(\left(\left(t^{2}+\mu^{2}\right) / 2\right) \cot \alpha-\mu t \csc \alpha\right)} & \alpha \neq n \pi \\
\delta(t-\mu) & \alpha=2 n \pi \\
\delta(t+\mu) & \alpha=(2 n \pm 1) \pi,\end{cases}
\end{aligned}
$$

where $\Gamma_{\alpha}=\sqrt{1-j \cot \alpha}$. In practical scenarios, discrete fractional Fourier transform (DFrFT) is used to deal with discrete received signals $[16,17]$. With DFrFT, (16) can be naturally represented as

$$
X_{\alpha}(u)=\Gamma_{\alpha} e^{j \pi u^{2} \cot \alpha} \int_{-\infty}^{\infty} e^{j 2 \pi \csc \alpha u t} e^{j \pi t^{2} \cot \alpha} x(t) d t
$$

By substituting (18) into (15), the observing matrix can be given as

$$
\mathbf{b}_{\alpha}(\mu)=\left[b_{\alpha}\left(\theta_{1}, \mu\right), \ldots, b_{\alpha}\left(\theta_{l}, \mu\right), \ldots, b_{\alpha}\left(\theta_{L}, \mu\right)\right] .
$$

\section{The Proposed Detection Method}

In the previous section, we transform the received signals at different sensors into a new vector which contains spatialtemporal and Doppler shift information of moving targets in the fractional Fourier domain. In this section, we utilize the Doppler shift characteristic of moving targets to propose 


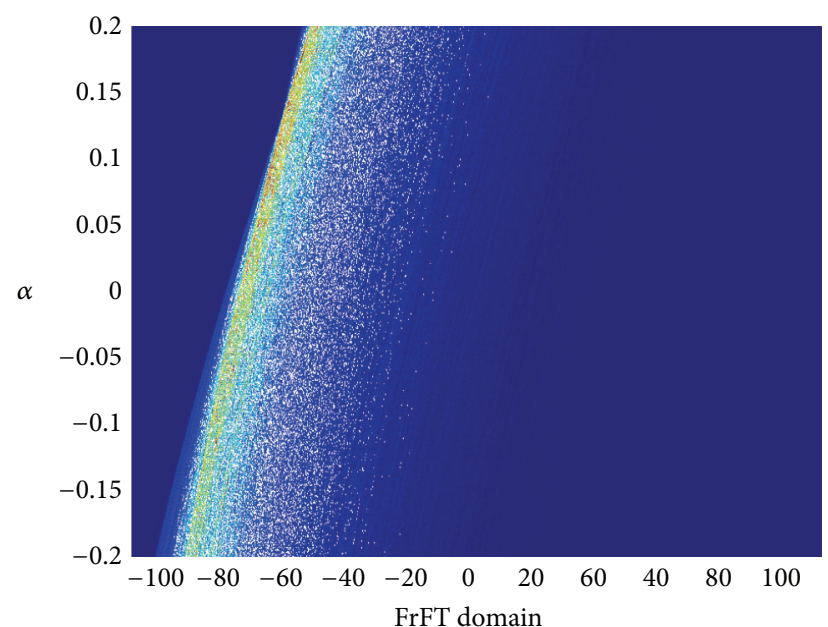

FIGURE 3: The fractional Fourier transform of received signal in SNR $=-15 \mathrm{~dB}$ and $\mathrm{SRR}=-15 \mathrm{~dB}$ environment.

a novel detection method in a severe reverberation environment.

In active sonar system, LFM signal as the transmitted signal can be written as

$$
y(t)=e^{j 2 \pi\left(\chi t^{2}+f_{0} t+\xi\right)},
$$

where $\chi$ is the rate, $f_{0}$ is the initial frequency, and $\xi$ is the initial phase. In discrete fractional Fourier transform, the optimal transform angle $\widetilde{\alpha}$ is dependent on rate $\chi$ of LFM signal. The interrelationship between rate and optimal angle is

$$
\widetilde{\alpha}=-\arctan \left(\frac{F_{s}^{2} / \Omega}{2 \chi}\right)
$$

where $\Omega$ is the total number of time samples and $F_{s}$ is the sampling rate. In practice, when we consider the moving targets, $\chi$ is varying due to Doppler shift effect. Hence, it is impossible to know the optimal transform angle in advance. Fortunately, an advantage of Doppler shift effect is that different target echoes have different $\chi$ values as well as optimal transform angles. Further, the energy of a signal is centralized on the certain LFM basis and its maximum amplitude will be at a particular $\mu$. Therefore, with the above two characteristics, the multiple target echoes can be conveniently separated in a nonreverberation environment [6]; consequently, it is easy to get the optimal $\widetilde{\alpha}$ and $\tilde{u}$ values in fractional Fourier domain [8].

However, the strong reverberation interference signal results in a low SRR as illustrated in Figure 3. In this case, using Doppler shift is not enough to detect targets. Therefore, we consider temporal and spatial information to detect multiple moving targets with Doppler shift information which makes the optimal transform angle $\alpha$ and location $\mu$ change in fractional Fourier domain [6]. When $\alpha_{p}$ and $\mu_{p}$ are the optimal transform angle and location of the $p$ th target in the $l_{p}$ subregion, we have

$$
b_{\alpha_{p}}\left(\theta_{l_{p}}, \mu_{p}\right) \gg b_{\alpha_{p}}\left(\theta_{l}, \mu_{p}\right), \quad \forall l_{p} \neq l
$$

This is illustrated using an example in Figure 4 with the plots of different $\mathbf{b}_{\alpha_{p}}\left(\mu_{j}\right)(j=1, \ldots, 6)$, where $\mu_{p}=\mu_{4}$ is the optimal value and $\theta_{l p}=\theta_{30}$ is the optimal direction. It is easy to observe that the fluctuation of curve $\mu_{p}=\mu_{4}$ is much more considerable than any other curve. In other words, the variance of vector $\mathbf{b}_{\alpha_{p}}\left(\mu_{p}\right)$ is most significant of all. Consequently, the variances of $\mathbf{b}$ can be used to measure the fluctuation degree by evaluating

$$
\begin{aligned}
& \mathbf{V}(\alpha, u)=\operatorname{var}\left\{\mathbf{b}_{\alpha}(u)\right\} \\
& \qquad u \in\left[-\frac{\sqrt{F_{s} T}}{2}, \frac{\sqrt{F_{s} T}}{2}\right], \alpha \in[-0.2,-0.1] .
\end{aligned}
$$

Figure 5 shows the simulation result of the variance calculation of all subregions. It can be clearly found that the target could be detected at the condition of low SRR and SNR. The number of targets is equal to the number of spikes. It means that the optimal $\left(\alpha_{p}, \mu_{p}\right)$ values of different targets can be acquired according to the locations of spikes.

\section{The Proposed Parameters Estimation Method}

In this section, we intend to develop a method for the estimation of velocity, range, and direction of arrival (DOA) with the knowledge of the number of targets and the optimal values $\left(\alpha_{p}, u_{p}\right)$ of each target.

5.1. Range Estimation. The transmitted signal with a rectangular window of duration $\Upsilon$ can be written as

$$
s(t)=y(t) \operatorname{rect}\left[\frac{(t-\Upsilon / 2)}{2}\right] \text {. }
$$

From (24), the received echo of the $p$ th target can be obtained as

$$
x_{p}(t)=s\left(\sigma_{p} t-\tau_{p}\right)
$$

where $\tau_{p}$ is the delay parameter and $\sigma_{p}$ is the compression/stretching parameter of the $p$ th target. By substituting (18) into (25), the received signal in fractional Fourier domain is expressed as

$$
X_{p}(u)=\Gamma_{\alpha} e^{j 2 \pi\left(\left(u^{2} / 2\right) \cot \alpha+\chi \tau_{p}^{2}-f_{0} \tau_{p}+\xi\right)} \int_{t_{2}}^{t_{1}} e^{j 2 \pi\left(\chi \sigma_{p}^{2}+(1 / 2) \cot \alpha\right) t^{2}} e^{j 2 \pi\left(f_{0} \sigma_{p}-2 \chi \sigma_{p} \tau-u \csc \alpha\right) t} d t
$$




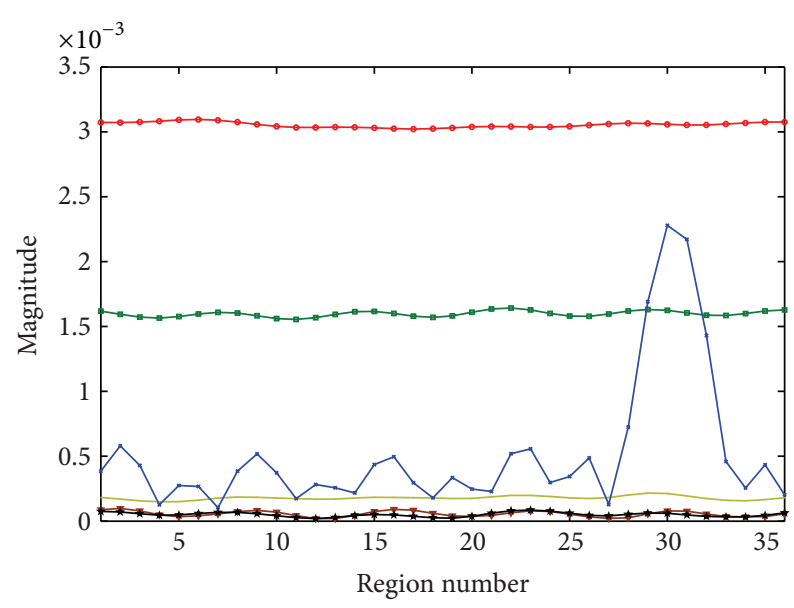

$$
\begin{array}{ll}
\rightarrow \mu_{1}=(-82.5162,-0.11) & \longrightarrow \mu_{4}=\mu_{p}=(28.8500,-0.11) \\
\rightarrow \mu_{2}=(-73.5718,-0.11) & \rightarrow \mu_{5}=(51.6490,-0.11) \\
-\mu_{3}=(10.5052,-0.11) & \rightarrow \mu_{6}=(64.6275,-0.11)
\end{array}
$$

FIGURE 4: The magnitudes of 6 curves of $\mathbf{b}_{\alpha_{p}}\left(\mu_{p}\right)$ in SNR $=-15 \mathrm{~dB}$ and $\mathrm{SRR}=-15 \mathrm{~dB}$.

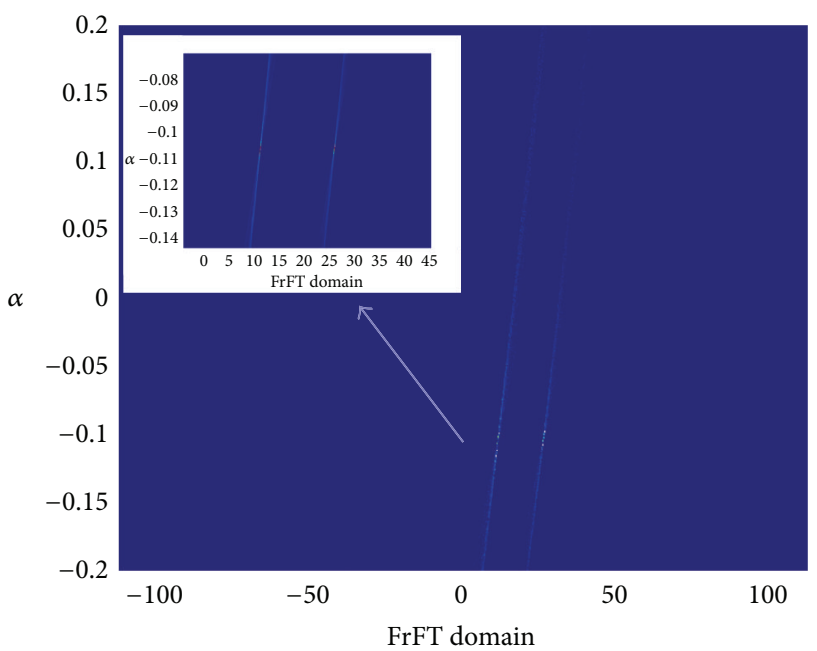

FIGURE 5: The variance values of different subregions in fractional Fourier domain. The environment is SNR $=-15 \mathrm{~dB}$ and SRR $=-15 \mathrm{~dB}$. There are two moving targets: one is located at $[1450,1550] \mathrm{m}$ with velocity being $[4,5] \mathrm{m} / \mathrm{s}$; the other is located at $[1600,1500] \mathrm{m}$ with velocity being $[5,4.5] \mathrm{m} / \mathrm{s}$.

where $t_{1}=(\Upsilon+\tau) / \sigma$ and $t_{2}=\tau / \sigma$, respectively. With (21) and optimal transform angle $\alpha_{p}$, the rate $\chi_{p}$ of the $p$ th target echo can be easily calculated by

$$
\chi_{p}=\frac{F_{s}^{2}}{2 \tan \left(-\alpha_{p}\right) \Omega} .
$$

It then follows that

$$
\sigma_{p}=\sqrt{\frac{\chi_{p}}{\chi}}
$$

According to [12], we can decompose $\int e^{j\left(p x^{2}+2 q x\right)} d x$ as

$$
\begin{aligned}
& \int e^{j\left(p x^{2}+2 q x\right)} d x \\
& =\sqrt{\frac{\pi}{2 p}} e^{-j\left(q^{2} / p\right)}\left[C\left(\frac{p x+q}{\sqrt{p}}\right)+j S\left(\frac{p x+q}{\sqrt{p}}\right)\right],
\end{aligned}
$$

where

$$
\begin{aligned}
& C(x)=\int_{0}^{x} \cos \left(\frac{\pi t^{2}}{2}\right) d t \\
& S(x)=\int_{0}^{x} \sin \left(\frac{\pi t^{2}}{2}\right) d t .
\end{aligned}
$$

Under (29) and (30), (26) can be rewritten as

$$
\begin{aligned}
& X_{p}(u) \\
& \quad=\Gamma_{\alpha} e^{j 2 \pi\left(\left(u^{2} / 2\right) \cot \alpha+\chi \tau^{2}-f_{0} \tau+\xi\right)} \sqrt{\frac{\pi}{2 h}} e^{-j\left(k^{2} / h\right)}\left[C\left(\frac{h t+k}{\sqrt{h}}\right)\right. \\
& \left.\quad+j S\left(\frac{h t+k}{\sqrt{h}}\right)\right]_{t_{2}}^{t_{1}},
\end{aligned}
$$

where $h=2 \pi\left(\chi \sigma_{p}^{2} \Omega / F_{s}^{2}+0.5 \cot \alpha\right), k=$ $\pi\left(f_{0} \kappa \sigma_{p}-2 \chi \kappa \sigma_{p} \tau_{p}-u \csc \alpha\right)$, and $g(u)=$ $e^{j \pi\left[\left(u^{2}\left(2 \chi \sigma_{p}^{2} \cot \alpha-1\right)+2 u \csc \alpha\left(f_{0} \sigma_{p}-2 \chi \sigma_{p} \tau_{p}\right)-\left(f_{0} \sigma-2 \chi \sigma_{p} \tau_{p}\right)^{2}\right) /\left(2 \chi \sigma_{p}^{2}+\cot \alpha\right)\right]}$ and $\kappa=\sqrt{T / F_{s}}$ is the scaling parameter.

Note that, in (31), the center values $\widetilde{\mu}$ of main lobe in the real part are determined by the Fresnel cosine integral $C(x)$, which crosses zeros only at $x=0$ as shown in Figure 6 . Therefore, we can get the zeros crossing, respectively, at

$$
\begin{aligned}
& \mu_{1}=\frac{h t_{1}+\pi \kappa \sigma_{p}\left(f_{0}-2 \chi \tau_{p}\right)}{\pi \csc \alpha_{p}}, \\
& \mu_{2}=\frac{h t_{2}+\pi \kappa \sigma_{p}\left(f_{0}-2 \chi \tau_{p}\right)}{\pi \csc \alpha_{p}} .
\end{aligned}
$$

As a consequence, we can get the center value $\widetilde{\mu}$ as

$$
\widetilde{\mu}=\mu_{p}=\frac{h\left(t_{1}+t_{2}\right)+2 \pi \kappa \sigma_{p}\left(f_{0}-2 \chi \tau_{p}\right)}{\pi \csc \alpha_{p}} .
$$

By substituting the estimates $\mu_{p}, \alpha_{p}$, and $\sigma_{p}$ into (34), the delay parameter of the $p$ th target can be obtained as

$$
\tau_{p}=\frac{2 \pi f_{0} \kappa \sigma_{p}-\pi \mu_{p} \csc \alpha_{p}+h\left(t_{1}+t_{2}\right)}{4 \pi \kappa \chi \sigma_{p}} .
$$

Furthermore, the $p$ th target distance $D_{p}$ is given as

$$
D_{p}=\tau_{p} \frac{c}{2} .
$$




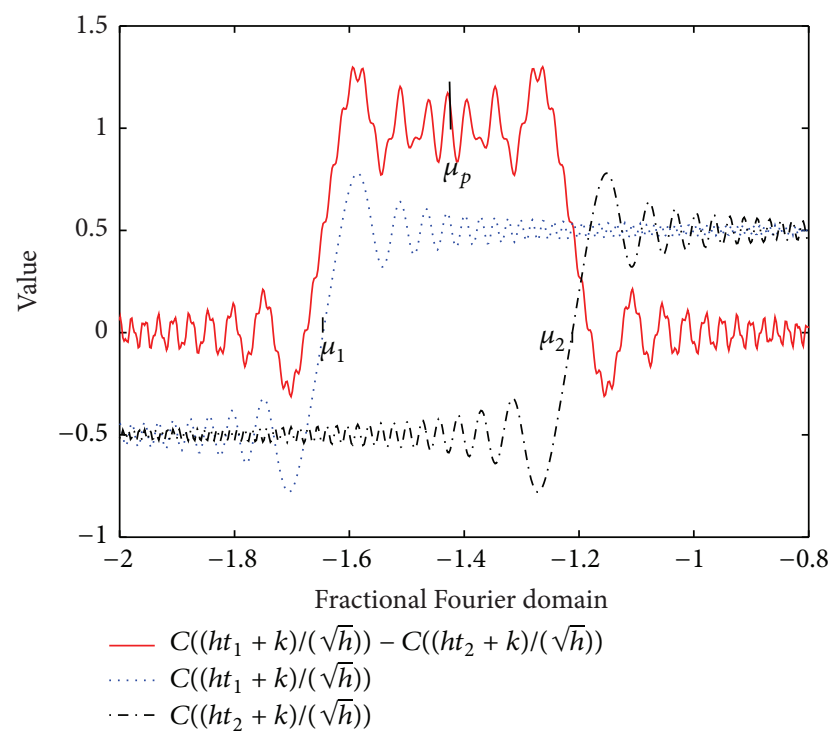

Figure 6: The main lobe formed by the subtraction of the two Fresnel cosine integrals in the FrFT of a received signal.

5.2. Direction of Arrival (DOA) Estimation. In this subsection, we propose a DOA estimation method using the optimal subregion $l_{p}$ and optimal values $\left(\alpha_{p}, u_{p}\right)$. By substituting (18) with the optimal values $\alpha_{p}$ and $\theta_{l_{p}}$ into (13), the subregion $l_{p}$ in the fractional Fourier domain can be written as $\widetilde{\mathbf{b}}_{\alpha_{p}}\left(\mu, \theta_{l_{p}}\right)$. Then, a bandpass filter with center frequency $\mu_{p}$ is applied to reduce the interference among the targets and effectively increase the SRR and SNR values. As a result, we can get a new vector $\widetilde{\mathbf{b}}_{\alpha_{p}, u_{p}}\left(\mu, \theta_{l_{p}}\right)$. Finally, utilizing the property of the FrFT, we can transform signal from $\widetilde{\mathbf{b}}_{\alpha_{p}, u_{p}}\left(\mu, \theta_{l_{p}}\right)$ to $\widetilde{\mathbf{b}}_{\alpha_{p}, u_{p}}\left(\omega, \theta_{l_{p}}\right)$.

As the transmitted signal is wideband, the truncation value varies along with frequency. We assume that the minimal truncation is $M_{1}=\left\lceil 2 \pi\left(f_{0}\right) / c R_{1}\right\rceil$ and the maximal truncation is $M_{2}=\left\lceil 2 \pi\left(f_{0}+B\right) / c R_{1}\right\rceil$, where $B$ is the bandwidth of the transmitted signal. That is to say, we have $M_{2}-M_{1}+1$ subbands. Each subband vector can be expressed as $\widetilde{\mathbf{b}}_{\alpha_{p}, u_{p}}^{m}\left(\omega, \theta_{l_{p}}\right)$, for $m=M_{1}, \ldots, M_{2}$, and the dimension of each subband vector is $(2 m+1) \times 1$. Then, according to [18], we can calculate the $p$ th target direction $\phi_{p}^{m}$ with the $m$ th frequency subband $\tilde{\mathbf{b}}_{\alpha_{p}, u_{p}}^{m}\left(\omega, \theta_{l_{p}}\right)$ of the $p$ th target. With the above development, the DOA of the $p$ th target can be obtained as

$$
\phi_{p}=\frac{1}{M_{2}-M_{1}+1} \sum_{m=M_{1}}^{M_{2}} \phi_{p}^{m} .
$$

\section{Simulation Results}

The numerical example considered here is an LFM signal as a transmitted signal, which swept from $2.1 \mathrm{kHz}$ to $3.05 \mathrm{kHz}$ over pulse duration of $0.1 \mathrm{~s}$. The initial phase is zero. Signal propagation speed is $1500 \mathrm{~m} / \mathrm{s}$. The sampling frequency $F_{s}$ equals $10 \mathrm{kHz}$. The total number of time samples is 80001 . The

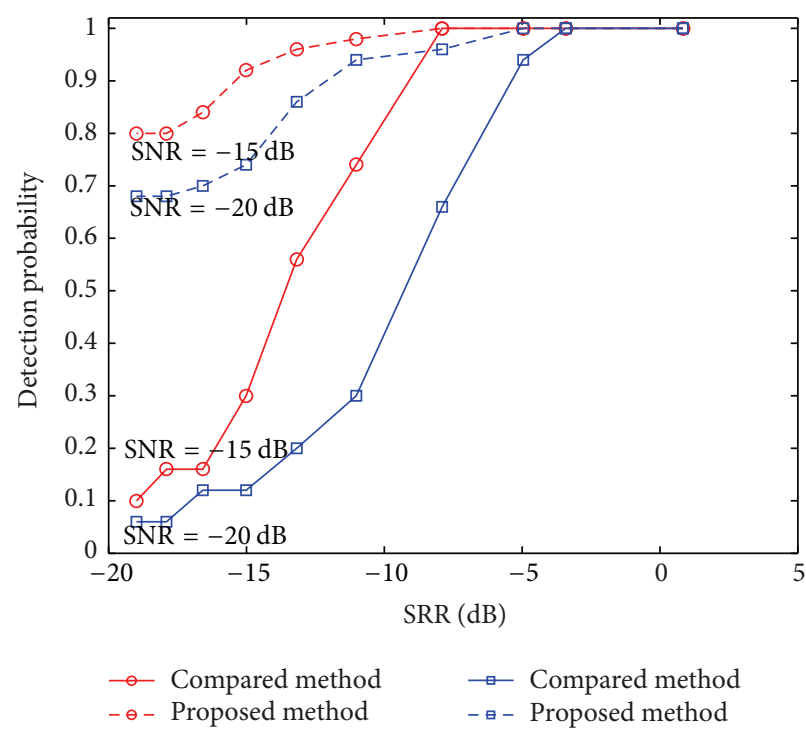

FIgURE 7: Detection probabilities with two detection methods over different SNR and SRR values.

bottom reverberation model is based on ray acoustics theory and follows the large number theorem [19]. The reverberation is assumed to be nonstationary and colored. The additive white Gaussian noise is zero-mean complex random process at each sensor.

In order to test the performances of multiple targets detection and parameters estimation, we consider two scenarios. Scenario I: we have one target which is located at $[1700,1500] \mathrm{m}$ in the Cartesian coordinates and moves with a radial velocity of $[4,5] \mathrm{m} / \mathrm{s}$. Scenario II: there are two adjacent targets. One is located at $[1500,1500] \mathrm{m}$; the other is located at $[1550,1500] \mathrm{m}$. The radial velocities of the two targets are $[5,5] \mathrm{m} / \mathrm{s}$ and $[4,4.5] \mathrm{m} / \mathrm{s}$ in different directions.

6.1. Detection Probability. Computer simulations are first carried out for the detection probability. In scenario I, the detection probability curves of the proposed method are illustrated in Figure 7, with the range of SRR being from $-20 \mathrm{~dB}$ to $3 \mathrm{~dB}$ and different SNR values. It can be seen that the proposed detection method performs well although in very low SRR scenario. In addition, the effect caused by additive Gaussian noise is less than that of reverberation.

Furthermore, we also compare the detection probability of the proposed method with the existing method in [6]. It can be observed from Figure 7 that both methods can get good probability detection in a high SRR environment. However, when the SRR value is less than $-10 \mathrm{~dB}$, the performance of the compared method declines sharply. Particularly, when the SRR is equivalent to $-11 \mathrm{~dB}$, it is hard to detect the target using the compared method. However, the proposed method still keeps a fine performance in an extremely low SRR environment. This is because the proposed method makes use of the differences of the Doppler shift between target echo and reverberation to detect target.

In scenario II, as shown in Figure 5, two adjacent targets can be easily distinguished. The simulation results which 


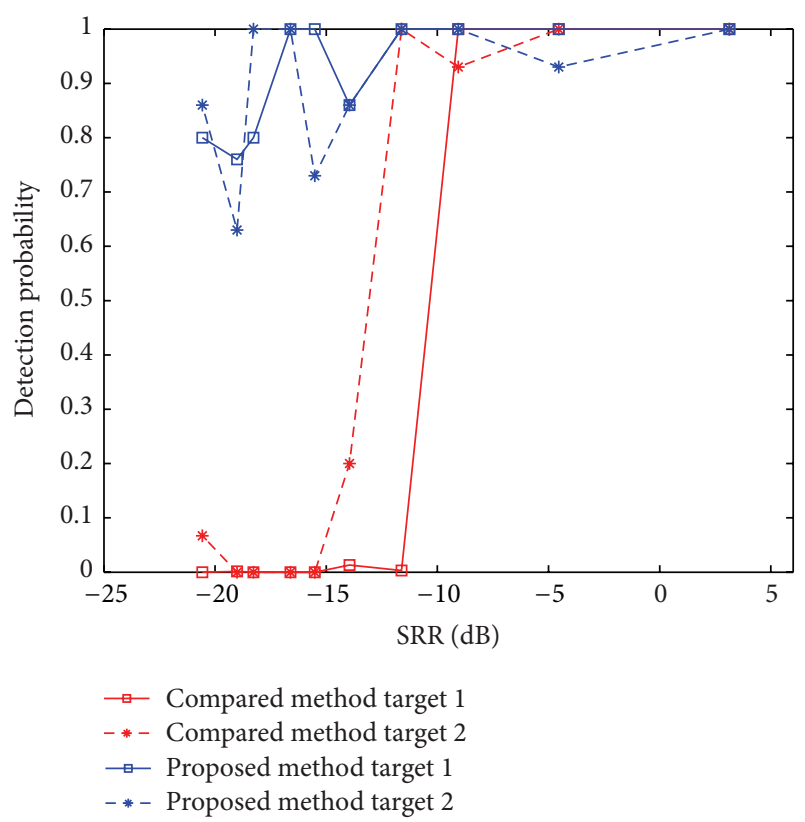

FIGURE 8: Detection probabilities of two targets with proposed detection methods over different SRR values and SNR is equal to $-15 \mathrm{~dB}$.

are presented in Figure 8 show that there is no mutual influence between the two targets by the proposed method and compared method. Specifically, the performance of the proposed detection method is better than the compared method in low SRR environment.

6.2. Parameters Estimation. In this subsection, parameters' (range and DOA) estimation of multiple targets simulation results are shown. Root-mean-square (RMS) error is used to measure the performance of the proposed method. The expression of RMS error is defined as

$$
\operatorname{RMS}(e)_{p}=\sqrt{\frac{1}{N} \sum_{n=1}^{N}\left|\hat{e}_{p}^{n}-e_{p}\right|^{2}},
$$

where $\widehat{e}_{p}^{n}$ is the $n$th estimation value of the $p$ th target and $e_{p}$ is the true value of the $p$ th target. $N$ is the total number of Monte Carlo runs. In the following simulations, $N$ is equal to 100 .

6.2.1. Range Estimation. In this subsection, we first consider scenario I to test the parameters estimation at low SRR values. Figure 9 shows the RMS range errors of single target with the SRR values varying from $-10 \mathrm{~dB}$ to $2 \mathrm{~dB}$. We can see that the range RMS errors decrease with some fluctuations along with the increase of SRR. This result from the main interference-reverberation has a great influence on the center of peaks, which determines the accuracy of parameters estimation. In consideration of the reverberation distribution being uniform [20], the effect caused by reverberation is random. Besides, we consider different environments with SNR chosen as $-10 \mathrm{~dB},-15 \mathrm{~dB}$, and $-20 \mathrm{~dB}$, respectively. Note

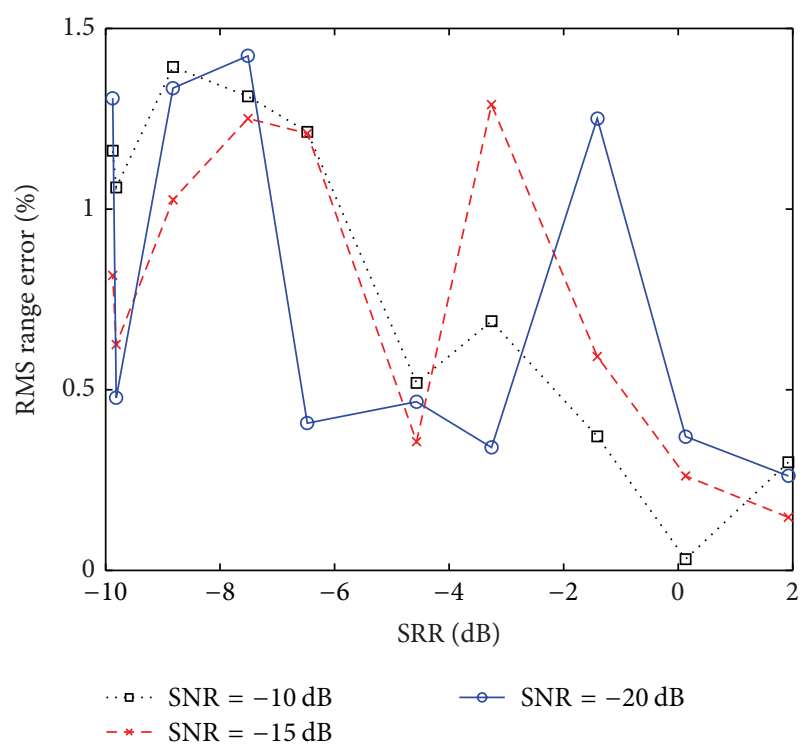

FIGURE 9: The RMS range errors of single target over different SNR and SRR values.

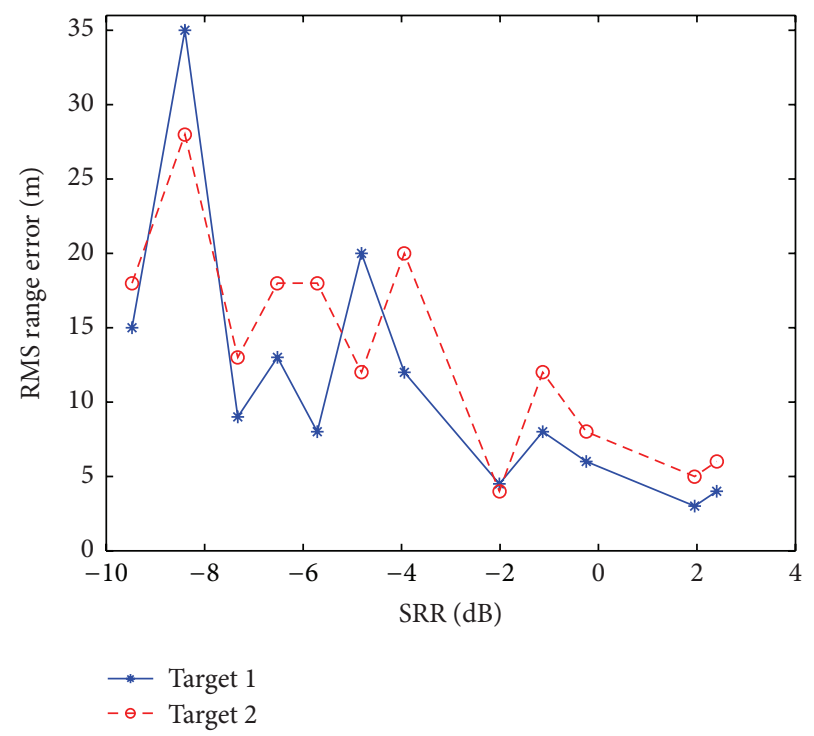

FIGURE 10: The RMS range errors of two targets over different SRR values and $\mathrm{SNR}$ is equal to $-15 \mathrm{~dB}$.

that the variation of SNR has a negligible effect on the accuracy of range estimation.

Figure 10 provides the simulation results in scenario II. Allowing for the accuracy of parameters is only determined by the widths of peaks. The proposed parameters' estimation method is not restricted and influenced by the number of targets.

6.2.2. DOA Estimation. Figure 11 displays the DOA errors in scenario I with different SNR and SRR values and compares the proposed DOA estimation method with the method given in [6]. The SRR varies from $-14 \mathrm{~dB}$ to $-2 \mathrm{~dB}$ and SNR is equivalent to $-10 \mathrm{~dB}$ and $-15 \mathrm{~dB}$. The proposed method maintains 


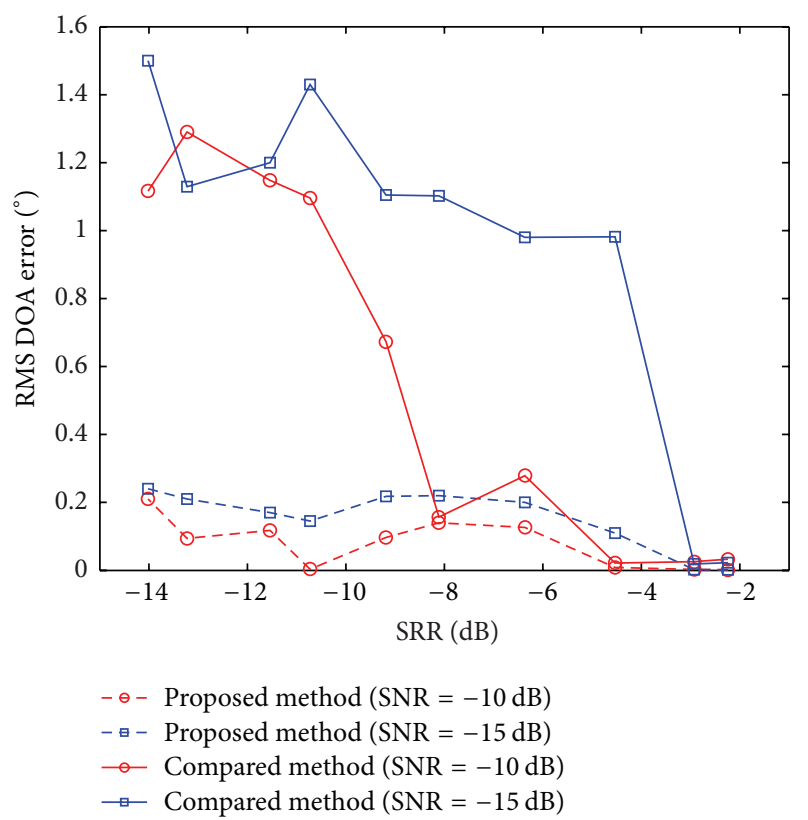

Figure 11: The RMS DOA errors with two methods over different SRR values and $S N R$ is equal to $-15 \mathrm{~dB}$.

good accuracy even in a low SRR environment. Also, the SNR has little impact on the accuracy. Both methods perform well in a high SRR environment. By using the beamforming to suppress reverberation, we can obtain high SRR values. This indicates that a higher accuracy can be guaranteed by the proposed method as compared to the method in [6], when considering a low SRR condition. In different SNR scenarios, the compared method has been more greatly affected than the proposed method. Furthermore, there are no interactions between these two targets due to the use of a bandpass filter to separate the received signals of multiple targets into $P$ subband in fractional Fourier domain.

\section{Conclusion}

In this paper, we proposed methods for detecting and estimating parameters (range and DOA) of multiple moving targets in a severe reverberation environment. Modal preprocessing, conventional beamforming, and fractional Fourier transform have been used to form a 3-dimensional matrix. With the properties of moving targets and reverberation, this matrix can be applied to detect multiple targets and estimate the parameters in strong reverberation environment. Specifically, the proposed scheme does not involve any a priori knowledge of environment parameters and number of targets. The simulation results demonstrate that the provided method can perform with high detection probabilities and maintain good accuracies of parameters estimation even in low SNR and SRR conditions. More importantly, the performances of the proposed method are not affected by the number of targets. The future work will focus on extending the method to detect and estimate parameters in a 3dimensional reverberation environment.

\section{Competing Interests}

The authors declare that there is no conflict of interests regarding the publication of this paper.

\section{References}

[1] G. Ginolhac and G. Jourdain, "'Principal component inverse' algorithm for detection in the presence of reverberation," IEEE Journal of Oceanic Engineering, vol. 27, no. 2, pp. 310-321, 2002.

[2] K. K. Cheepurupalli and R. R. Konduri, "Noisy reverberation suppression using adaboost based EMD in underwater scenario," International Journal of Oceanography, vol. 2014, Article ID 563780, 7 pages, 2014.

[3] A. B. Baggeroer, W. A. Kuperman, and P. N. Mikhalevsky, "An overview of matched field methods in ocean acoustics," IEEE Journal of Oceanic Engineering, vol. 18, no. 4, pp. 401-424, 1993.

[4] W. Xu, Z. Xiao, and L. Yu, "Performance analysis of matchedfield source localization under spatially correlated noise field," IEEE Journal of Oceanic Engineering, vol. 36, no. 2, pp. 273-284, 2011.

[5] P. A. Forero and P. A. Baxley, "Shallow-water sparsity-cognizant source-location mapping," Journal of the Acoustical Society of America, vol. 135, no. 6, pp. 3483-3501, 2014.

[6] P. H. Leong, T. D. Abhayapala, and T. A. Lamahewa, "Multiple target localization using wideband echo chirp signals," IEEE Transactions on Signal Processing, vol. 61, no. 16, pp. 4077-4089, 2013.

[7] E. Sejdić, I. Djurović, and L. Stanković, "Fractional Fourier transform as a signal processing tool: an overview of recent developments," Signal Processing, vol. 91, no. 6, pp. 1351-1369, 2011.

[8] Y. Ma and Y. Kong, "FRFT based on joint estimation time delay and radial velocity of underwater target," in Proceedings of the 3 rd International Congress on Image and Signal Processing (CISP '10), vol. 9, pp. 4074-4078, IEEE, Yantai, China, October 2010.

[9] A. Kalgan, R. Bahl, and A. Kumar, "Source ranging and direction of arrival estimation using accelerometer based implementation of prototype acoustic vector sensor," in Proceedings of the IEEE Underwater Technology (UT '15), Chennai, India, February 2015.

[10] P. Palanisamy, N. Kalyanasundaram, and P. M. Swetha, "Twodimensional DOA estimation of coherent signals using acoustic vector sensor array," Signal Processing, vol. 92, no. 1, pp. 19-28, 2012.

[11] Y. Song and K. T. Wong, "Azimuth-elevation direction finding using one four-component acoustic vector-sensor spread spatially along a straight line," The Journal of the Acoustical Society of America, vol. 137, no. 4, p. 2387, 2015.

[12] D. Zwillinger, Table of Integrals, Series, and Products, Elsevier, New York, NY, USA, 2014.

[13] D. B. Ward and T. D. Abhayapala, "Reproduction of a planewave sound field using an array of loudspeakers," IEEE Transactions on Speech and Audio Processing, vol. 9, no. 6, pp. 697-707, 2001.

[14] T. D. Abhayapala, R. A. Kennedy, and R. C. Williamson, "Nearfield broadband array design using a radially invariant modal expansion," Journal of the Acoustical Society of America, vol. 107, no. 1, pp. 392-403, 2000. 
[15] H. Sun, S. Yan, and U. P. Svensson, "Robust minimum sidelobe beamforming for spherical microphone arrays," IEEE Transactions on Audio, Speech and Language Processing, vol. 19, no. 4, pp. 1045-1051, 2011.

[16] H. M. Ozaktas, O. Ankan, M. Alper Kutay, and G. Bozdagi, "Digital computation of the fractional fourier transform," IEEE Transactions on Signal Processing, vol. 44, no. 9, pp. 2141-2150, 1996.

[17] A. Bultheel, "A two-phase implementation of the fractional fourier transform," TW Reports, 2011.

[18] R. O. Schmidt, "Multiple emitter location and signal parameter estimation," IEEE Transactions on Antennas and Propagation, vol. 34, no. 3, pp. 276-280, 1986.

[19] P. C. Etter, Underwater Acoustic Modeling and Simulation, CRC Press, 4th edition, 2013.

[20] J. J. Murray, "A theoretical model of linearly filtered reverberation for pulsed active sonar in shallow water," Journal of the Acoustical Society of America, vol. 136, no. 5, pp. 2523-2531, 2014. 


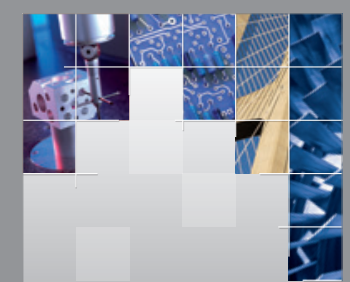

\section{Enfincering}
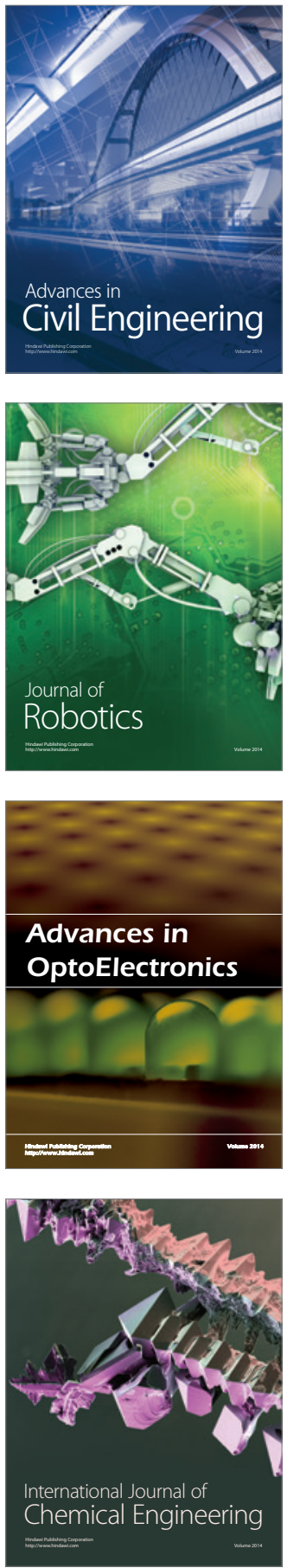

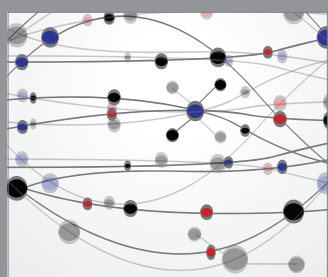

The Scientific World Journal

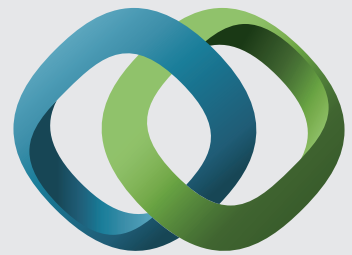

\section{Hindawi}

Submit your manuscripts at

http://www.hindawi.com
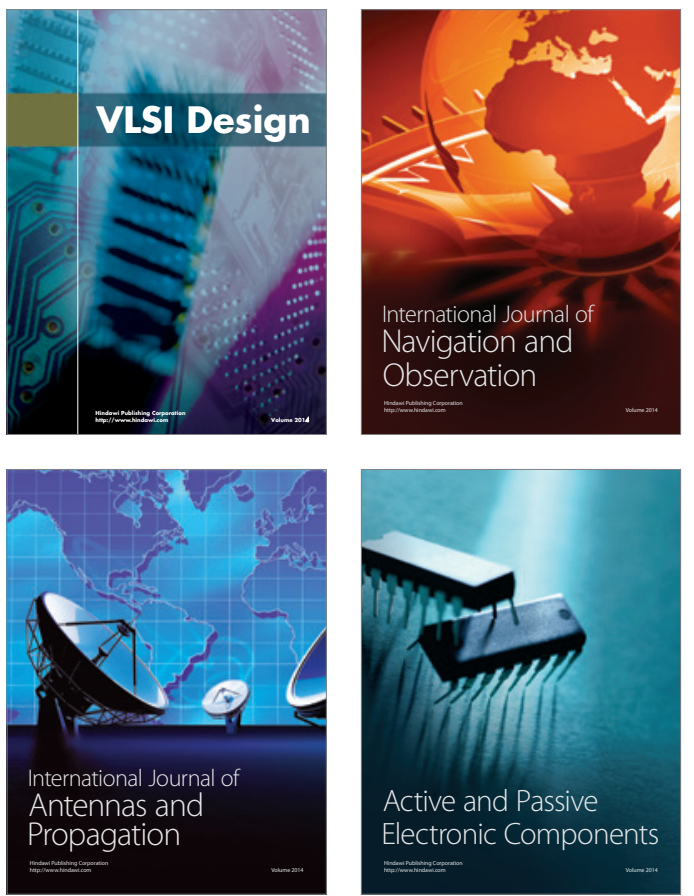
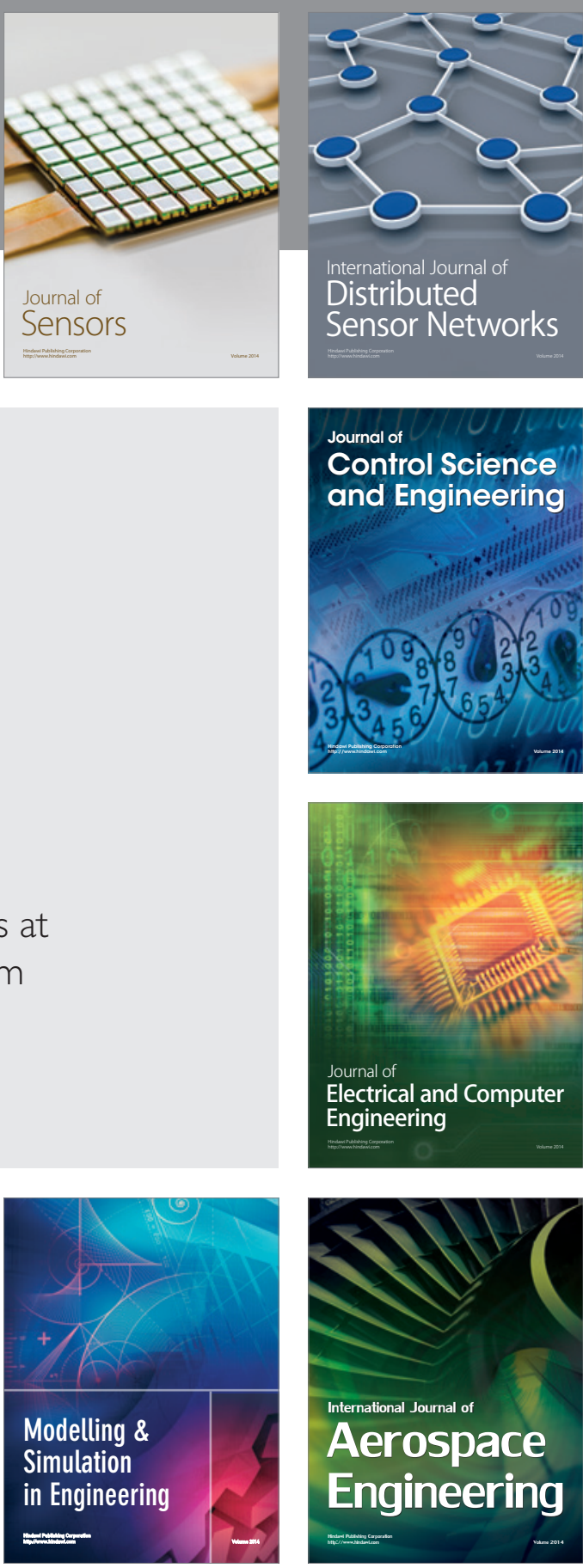

International Journal of

Distributed

Sensor Networks

Journal of

Control Science

and Engineering
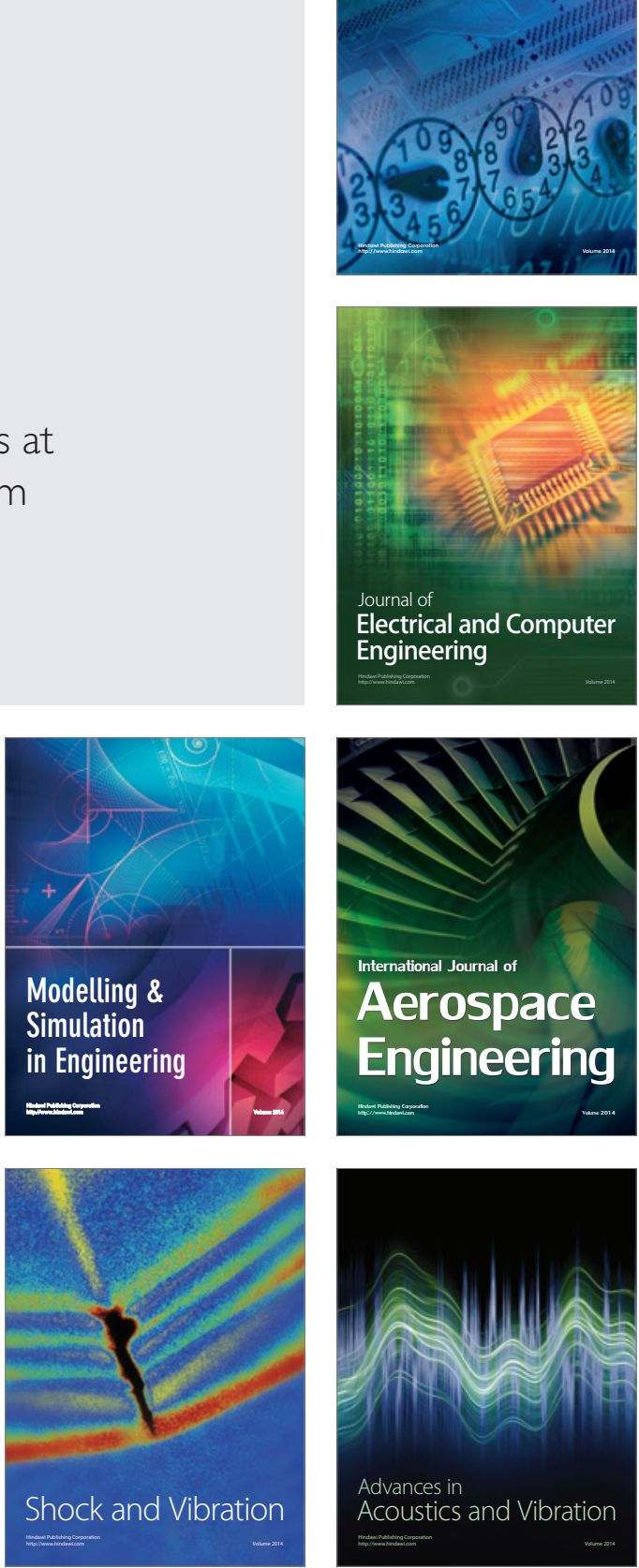